\title{
Philosophiques
}

\section{Réponses des auteurs}

\section{G. B. Madison et Th. F. Geraets}

Volume 2, numéro 1, avril 1975

URI : https://id.erudit.org/iderudit/203026ar

DOI : https://doi.org/10.7202/203026ar

Aller au sommaire du numéro

Éditeur(s)

Société de philosophie du Québec

ISSN

0316-2923 (imprimé)

1492-1391 (numérique)

Découvrir la revue

Citer ce document

Madison, G. B. \& Geraets, T. F. (1975). Réponses des auteurs. Philosophiques,

2(1), 124-130. https://doi.org/10.7202/203026ar

Ce document est protégé par la loi sur le droit d'auteur. L'utilisation des services d'Érudit (y compris la reproduction) est assujettie à sa politique d'utilisation que vous pouvez consulter en ligne.

https://apropos.erudit.org/fr/usagers/politique-dutilisation/ 


\section{RÉPONSE A Th. F. GERAETS \\ par G. B. Madison}

Comme Geraets l'indique, j’ai proposé dans mon livre d'opérer une «reprise interprétante» plutôt qu'une «présentation» ou un «commentaire» de l'oeuvre de Merleau-Ponty. C'est dire que mon étude n'est pas «historique» dans le sens habituel du terme, une tentative de montrer comment l'oeuvre de Merleau-Ponty s'est constituée au fur et à mesure des années. Je n'ai pas voulu me projeter en arrière et revivre la philosophie de Merleau-Ponty telle qu'il l'a lui-même vécue, mais l'examiner du point de vue auquel nous nous situons aujourd'hui. Mon intérêt était donc plutôt de nature critique que documentaire ou historique. C'est ainsi que je ne voulais pas me limiter uniquement à l'interprétation que Merleau-Ponty faisait lui-même de son oeuvre. Cela aurait été prendre les intentions de l'auteur comme la seule et adéquate indication du sens et de la valeur de 
ce qu'il a effectivement réalisé. Or, je crois qu'il existe souvent un décalage entre ce qu'on croit faire et ce qu'on fait réellement, que la compréhension de soi qu'on a de soi-même est souvent trompeuse et, en un mot, que les bonnes intentions ne font pas nécessairement des bonnes oeuvres. Ce qui m'intéressait donc, ce n'était pas tellement la visée consciente de Merleau-Ponty dans la PP telle qu'il l'a lui-même vécue et comprise que la question de l'adéquation de cette oeuvre à la réalité des choses. Et ce que j'ai cru trouver, c'est que la PP est inadéquate et pour cette raison que Merleau-Ponty n'y avait pas encore suffisamment rompu avec la philosophie de la conscience, avec l'idéalisme traditionnel.

C'est cette thèse qui semble constituer un point de désaccord entre Geraets et moi-même. Geraets aurait plutôt tendance à dire que la PP accomplit déjà un pas décisif dans le dépassement de la philosophie de la conscience. Mais cette différence est peut-être plus apparente que réelle et tient à nos façons d'approcher Merleau-Ponty plutôt qu'à ce que nous y trouvons. Outre cette question de la «première philosophie », Geraets signale deux autres domaines où il $\mathrm{y}$ aurait entre nous une différence d'interprétation : l'attitude de Merleau-Ponty vis-à-vis Husserl et la notion de l'Être dans les derniers écrits. Comme je ne peux pas dans le temps donné répondre en détail à chacun de ces points, je vais tenter de les traiter tous les trois ensemble, mais d'une façon oblique, en prenant comme thème la question du langage philosophique. Je pourrais peut-être de la sorte non seulement répondre à la substance des remarques de Geraets mais aussi élucider davantage le sens de nos divergences et peutêtre même dissiper certains malentendus possibles.

Une chose que nous a enseignée Merleau-Ponty, c'est qu'il existe entre la pensée et la parole un rapport étroit et indissoluble. Le langage n'est pas le simple véhicule d'une pensée autonome. Même si par un certain côté la pensée dépassait son expression, c'est par l'expression que ce dépassement est rendu possible. Cela veut dire par conséquent que le sens de ce qu'on dit ne peut rester indifférent à la façon dont on le dit ou, autrement dit, que les expressions et les mots qu'on utilise déterminent pour une grande part la signification de ce qu'on dit, quel que 
soit par ailleurs le sens qu'on croit exprimer en le disant. C'est ainsi, par exemple, qu'une critique de la technologie qui se voudrait radicale et qui cependant n'emploierait que des termes et des concepts technologique, échouerait comme critique et continuerait à jouer le jeu de la technologie. Et c'est justement pour cette raison que je soutiens que la PP échoue dans sa tentative de dépasser l'intellectualisme et n'enseigne en fin de compte qu'une mauvaise ambiguïté. Je suis complètement en accord ave: Geraets quand il soutient que la véritable visée de Merleau-Ponty dans la PP est de rompre avec les catégories traditionnelles de l'idéalisme. Seulement, j'estime que Merleau-Ponty n'a pas réussi cette tentative à cause des concepts-clés qu'il y emploie, telle la notion husserlienne d'intentionnalité qui est finalement l'expression d'une philosophie idéaliste. Et c'est pour cela que je parle d'une mauvaise ambiguïté, car dans la PP l'intention de l'auteur et la signification réelle de l'oeuvre ne se recouvrent pas. Peutêtre que la différence entre Geraets et moi tient en grande partie à ce que Geraets est prêt à accorder plus de considération aux intentions de l'auteur que moi, l'oeuvre même m'intéressant plus que l'auteur.

Certes Geraets est prêt à accorder que le langage de la PP est effectivement «idéaliste»: «la langue philosophique de la PP est celle d'une philosophie de la conscience. Tout ce système linguistique est articulé autour de la distinction radicale 'conscience'-'objet'...» Seulement il ajoute: «... mais l'usage que Merleau-Ponty fait de cette langue vise justement à remettre en question cette distinction même». N'empêche qu'à cause de sa conceptualité idéaliste, le projet de la $\mathrm{PP}$ — et c'est Geraets qui le dit - «devrait rester inadéquat». La différence entre nous semble donc être que Geraets veut parler d'inadéquation alors que je préfère parler tout simplement d'échec. Mais Geraets admet lui-même: «Ce n'est pas négligeable, surtout pour une philosophie qui insiste tellement sur l'unité de la pensée et de la parole...»».

Quand il s'agit d'un penseur aussi fécond que Merleau-Ponty il existe effectivement une marge de liberté permettant aux interprètes de mettre l'accent différemment. Puisque Geraets estime que «depuis Kant on ne saurait plus concevoir une philosophie, 
digne de ce nom, qui ne serait plus transcendentale », il essaie de montrer comment Merleau-Ponty a réalisé une nouvelle philosophie transcendentale libérée des préjugés d'une philosophie de la conscience. Mais puisque j'estime pour ma part que philosophie transcendentale, philosophie de la conscience et idéalisme sont tous étroitement liés entre eux et qu'une philosophie de l'expérience telle que nous la présente la PP opère avec les mêmes présuppositions de base qu'une philosophie de la conscience plus traditionnelle, je ne peux voir dans la $\mathrm{PP}$, en dernière analyse, qu'un échec. Le livre est un échec, car ce que MerleauPonty semble vouloir y dire ne peut tout simplement pas être dit dans le langage et avec la conceptualité auxquels il a recours et qui sont ceux de la tradition. Comme l'a remarqué Granel :

il reste à savoir si les mots ne sont que des mots, qu'on puisse habiller de tei et tel sens, comme les petites filles croient chancer de poupúe en changeant leur poupée; ou si les mots sont touiours les mots d'un certain langage, investis par le sens qui s'investit en eux et auquel ils réduisent implacablement toute signification que l'on prétend leur donner de l'extérieur. (Le sens du temps et de la perception cbez E. Husserl, p. 106).

Il est donc d'autant plus significatif que quand dans $L e$ visible et l'invisible Merleau-Ponty essaie de réaliser « un nouveau point de départ», il essaie aussi, et cela expressément, de rompre avec la terminologie de la philosophie traditionnelie. Quand il dit qu'il faut «tout reprendre » (VI, 172), il veut dire qu'il faut rompre avec les concepts traditionnels - tels que sujet, objet, existence, essence - et former des «nouveaux instruments » linguistiques et conceptuels. Il écrit :

... nous nous interdisons... d'introduire dans notre description les concepts issus de la réflexion, qu'elle soit psychologique ou transcendentale... Il nous faut renoncer, en commençant, à des notions telles que «actes de conscience», « états de conscience », «matière », «forme », et même «image » et «perception». (VI, 209).

Cela devrait suffire pour montrer que le dernier Merleau-Ponty était pleinement conscient des difficultés du langage et voulaic alors les surmonter. Ce qui est d'ailleurs du plus grand intérế 
pour nous aujourd'hui dans l'oeuvre finale de Merleau-Ponty, c'est la façon dont il soulève la question du discours philosophique. C'est en réalité toute la question du statut du discours philosophique qui se trouve mise en position centrale quand il écrit :

C'est donc une question de savoir si la philosophie comme reconquête de l'être brut ou sauvage peut s'accomplir par les moyens du langage éloquent, ou sil ne lui faudrait pas en faire un usage qui lui ôte sa puissance de signification immédiate ou directe pour l'égaler à ce qu'elle veut tout de même dire. (VI, 139)

Cependant, et je le répète, le nouveau point de départ du dernier Merleau-Ponty n'est pas un Kebre heideggerien, une simple coupure ou rupture avec la tradition (encore qu'on pourrait se demander si Heidegger est aussi radical qu'il pense être). C'est, à l'encontre de Heidegger, à partir de la conscience, de la subjectivité, de Husserl et de la tradition que Merleau-Ponty, lui, s'interroge, et d'une manière radicale. C'est dire que Merleau-Ponty n'a jamais voulu tout simplement abandonner Husserl; il voulait dépasser la philosophie transcendentale, mais pour ainsi dire de l'intérieur. Je peux donc accepter pleinement les propos de Geraets quand il dit que «c'est à travers la décentration, la déformation cohérente de la langue husserlienne que s'enseigne peu à peu l'intention significative du discours de Merleau-Ponty ». Seulement j'ajouterais que cette déformation finit par tout remettre en question, que l'\& excès du vécu » finit par briser les cadres du transcendentalisme et de l'immanentisme (la réduction de tout être à la conscience, à l'expérience) qui en découle. Une phénoménologie qui pense résolument ses propres limites a déjà dépassé la phénoménologie classique, et peut-être même la tradition. .

Tout cela nous amène à la notion de l'Être dans les derniers écrits. Je me limiterai cependant à des remarques suivantes. Le terme «Être » est ce qu'il y a de plus traditionnel - et de plus équivoque. Merleau-Ponty, il est vrai, l'utilise dans sa dernière oeuvre, mais il emploie aussi un autre terme - la chair - et comme il le dit: "on sait qu'il n'y a pas de nom en philosophie traditionnelle pour désigner cela ». (VI, 183). C'est cet «Être» 
qui ne se réduit pas à l'être-au-monde, c'est-à-dire à la relation sujet-monde, à l'«expérience », qui permet à Merleau-Ponty de dépasser la contingence. Mais ce dépassement de la contingence n'est pas un retour à la nécessité, car l' «Être » ici n'est ni un être suprême et nécessaire (ens causa sui, ens realissimum) ni un principe logique transcendental (un «ce sans quoi» rien n'est pensable). Il n'a de réalité ni logique ni ontique; ce n'est pas tant un fondement, donc, qu'un abîme, une source insondable et inépuisable de transcendance. Cet «Être » qui échappe à l'ordre de l'essence échappe du même coup au couple conceptuel contingence/nécessité (alors qu'un rationaliste comme Husserl conçoit le premier terme de ce couple comme étant subordonné au deuxième, le jeune Merleau-Ponty, anti-rationaliste, se contentait d'en renverser seulement la priorité). Au-delà de toute essence, il est aussi, en fin de compte, au-delà du langage et ne peut être saisi qu'indirectement, dans les interstices de la parole des hommes.

\section{RÉPONSE A G. B. MADISON}

par Th. F. Geraets

Je suis étonné de voir que Madison dédlare que son étude de Merleau-Ponty «n'est pas 'historique' dans le sens habituel du terme ». N'est-ce pas vouloir faire un travail d'historien que «de surprendre et de suivre la genèse de l'ontologie de Merleau-Ponty...» (p. 18)? En effet, je ne pense pas que Madison, quand il qualifie son livre de « reprise interprétative» l'entend dans le sens où le livre de Heidegger sur Kant peut être appelé ainsi. Le sens que prend le terme dans le cas de Madison semble plus proche de ce qu'on appelle normalement « historique », même s'il s'agit surtout de l'histoire intérieure de l'oeuvre, plutôt que des facteurs extérieurs qui ont influencé son développement.

Madison a raison d'insister sur la nécessité de ne pas s'en tenir à l'interprétation explicite que Merleau-Ponty donne lui- 
même de son oeuvre, à ses intentions exprimées de façon programmatique. Il faut étudier ce que l'auteur «a effectivement réalisé », c'est-à-dire ce qu'il a réussi à exprimer. Selon moi, ce que Merleau-Ponty a effectivement exprimé dans la Pbénoménologie de la perception constitue un dépassement véritable de la philosophie transcendantale traditionnelle. Mais en rompant avec la présupposition traditionnelle du primat du sujet, et en commençant ainsi à développer une nouvelle philosophie, il tenait encore à la qualifier de transcendantale. Ce n'est pas en mon propre nom que j'ai écrit que «depuis Kant on ne saurait plus concevoir une philosophie, digne de ce nom, qui ne serait plus transcendantale» (mon livre, p. 87). C'est ce que pensait Merleau-Ponty en écrivant La structure du comportement (Ibid., $\mathrm{n}$. 46), et ce que, dans un sens nouveau qui commençait à s'affirmer dans la Phénoménologie de la perception, il a maintenu jusqu'à la fin (cf. VI 226, cf. ici p. 110).

Je n'entends nullement opposer intention et expression effective, dans le sens où je serais intéressé davantage par les intentions de l'auteur que par son oeuvre même. Mais il me semble que la signification réelle de la Pbénoménologie recouvre déjà l'intention anti-idéaliste: la décentration de la langue husserlienne, - décentration que Madison admet, - est telle qu'elle brise déjà « les cadres du transcendantalisme et de l'immanentisme », qu'il s'y dégage une nouvelle notion de l'expérience qui ne résulte pas d'une simple désintellectualisation de la conscience.

Peut-on dire que «cette déformation finit par tout remettre en question »? Il conviendrait de distinguer entre les aspects de la pensée husserlienne que Merleau-Ponty a effectivement mis en question et d'autres dont il s'est inspiré jusqu'à la fin. Et il faudrait déterminer le moment de la mise en question. Faire ceci est impossible dans le cadre de cette brève discussion. Revenons, toutefois, un instant à la notion d'intentionnalité. Ou bien la thèse de Madison que cette «notion husserlienne... est finalement l'expression d'une philosophie idéaliste» vaut pour toute intentionnalité, et dans ce cas le dernier Merleau-Ponty est encore idéaliste. Ou bien elle ne s'applique pas à l'intentionnalité opérante, et alors le Merleau-Ponty de la Pbénoménologie n'est déjà plus idéaliste. 
Que le dépassement de la philosophie transcendantale traditionnelle se fait d'abord (et, dans une certaine mesure jusqu'à la fin) par une parole qui décentre la langue husserlienne, - estce que cela nous autorise à parler d'échec? Il me semble que c'est un pas très réel dans la bonne direction, appelant des explicitations ultérieures, mais qui est beaucoup plus qu'une simple déclaration d'intention. Je ne crois pas que l'on peut opposer, dans la mesure où Madison semble le faire, ce que Merleau-Ponty croyait faire et ce qu'il a fait réellement. 